\title{
Family vs. Individual Profiles in a Health Portal: Strengths and Weaknesses
}

\author{
Nathalie Colineau and Cécile Paris \\ CSIRO - ICT Centre \\ PO Box76, Epping NSW 1710 \\ Australia \\ firstname.lastname@csiro.au
}

\begin{abstract}
Our research aims at helping families work together to achieve a healthier lifestyle, increasing their awareness of what they currently do and what they could do differently. We built a prototype collaborative platform for families to see if we could facilitate health discussions and encourage supportive behaviour within the family. This paper presents a trial we conducted online where we investigated what would be more promising: an individual profile, space and goal, for each individual in a family, or a family profile, space and goal. We show that both conditions have their strengths and weaknesses: whereas a specific goal at the individual level tends to lead to better task performance, a shared goal seemed to promote support within the family. Similarly, whereas individual spaces seemed to encourage more personal reflections, the shared space attracted more non-task related visits to the portal by family members.
\end{abstract}

Family Support, Health Behaviour, User Profiles, Wellbeing, Evaluation.

\section{INTRODUCTION}

Obesity is a concern worldwide with an increasing proportion of people overweight or obese. Research has focused on preventive programs with numerous health behaviour change interventions (e.g. Hardeman et al., 2000 and Noar et al., 2007), and the development of technologies promoting healthy living and supporting behaviour change (e.g. Consolvo et al., 2008 and Mattila et al., 2008). Interventions and well-being applications are typically based on psychological models and behavioural theories. They aim to deliver effective health communication and to provide support and encouragement to impact people's behaviour.

Most of this effort, however, has focused on individuals, with little work involving families, and even less technology built to facilitate and support health discussions within families. Research on social integration and social support has shown that people's natural social context can influence people's behaviour, and, in particular, their health and well-being, through the nature and the amount of social support provided (for a review see Verheijden et al., 2005). Research into family influence has also shown that a supportive environment within the family can promote health behaviour change and improve compliance with medical treatments. -...a patient diagnosed with Type I/ diabetes may be more likely to change his diet and exercise patterns if his wife and children support these changes and if the family collectively followed through with a plan to establish a healthier lifestyle" (Jones et al., 2004; p.636).

Our aim is to explore the role technology can play in supporting reflection and sharing by building an environment that supports families in their journey towards a healthier lifestyle, encouraging them to discuss, reflect and work together towards that goal. We built an initial prototype to experiment with various strategies for engaging families towards a healthy lifestyle, facilitating reflections upon their current lifestyle. In particular, we wanted to investigate the respective advantages and disadvantages of providing an individual profile, space and goal, for each individual in a family, and a family profile, space and goal.

In this paper, we present the results of a two-week field trial we conducted with eighteen families of four (i.e. two parents and two children aged from 12 to 17 , living in the same household), focusing on two conditions: one in which the environment was totally family oriented, and one in which it was individual oriented. We start with a discussion of research on family-based interventions and the common strategies used for health behaviour change. We present the technology probe we built, the trial we conducted and our results. Finally, we discuss what we learnt and conclude. 


\section{RELATED WORK}

We briefly present research conducted within a family context and work promoting health discussions and reflections.

\subsection{Family-based interventions}

Although a lot of effort has been deployed in health promotion, targeting children in particular, there has been little work involving families. Most of the family based interventions do not systematically involve the whole family (McLean et al., 2003): some only involve spouses; others target a parent with a child. Because each member potentially influences other family members without necessarily having the ability to control their behaviour (De Bourdeaudhuij, 1997), it is difficult to know which family member to target. Nutrition interventions have been directed to almost all family members involving various strategies with mixed success. Mothers have been particularly targeted by researchers. Often in charge of the shopping and cooking, they are perceived as the ones responsible for the family diet and thus able to influence the family behaviour. Children and adolescents, also often considered as able to influence the whole family, have been targeted through school-based interventions. Despites all this effort, the most appropriate way to involve family members is still an open question. Similarly, there has been little research done in the design of preventive health applications for families. $\mathrm{HCl}$ research on wellbeing and fitness applications have been almost exclusively designed for individuals, with a focus on mobile applications (e.g. Consolvo et al., 2008, Toscos and Connelly, 2008). It is worth noting however, the field study done by Grimes and colleagues (Grimes et al., 2009) with families, where they investigated issues related to the collection and sharing of health information within the family context.

\subsection{Reflective thinking in health}

Facilitating health discussions and encouraging people to reflect on their health, without necessarily asking them to modify their behaviour, has been studied to some extent. For example, EatWell (Grimes et al., 2008) was designed for people to create and share nutrition-related memories. It demonstrated how the crafting of emotionally and culturally charged stories could motivate people to follow health ideas from others. FotoFit (Brown et al., 2006) was designed to raise student's awareness of their diet and exercise patterns. It showed that a photo-based food journal was a useful tool to support reflection and activity assessment. Finally, MAHI (Mamykina et al., 2008) was developed to encourage reflective thinking skills through interactions between individuals recently diagnosed with diabetes and their educator. It illustrated how a health monitoring application could influence people's attitude and behaviour.

Building on this body of research, we looked at ways to make family members work together towards a healthier lifestyle, with a particular emphasis on encouraging reflections and discussions within a family context.

\section{ENGAGING THE WHOLE FAMILY IN HEALTHY LIVING}

The platform we built was based on an initial set of requirements collected through a survey (Colineau et al., 2009). This first prototype, the family portal to promote healthy living, was used as an evaluation platform to conduct the field study reported here. We implemented different versions to suit our experimental needs. The common version of the portal was designed around four sections: 1) a message board, allowing family members to leave messages for each other and providing the system with a means to send reminders and recommendations; 2) a lifestyle ideas tool, enabling family members to record their current lifestyle and think of how they could improve it; 3) a health section, providing a set of resources about healthy lifestyle and wellbeing; and 4) a diary tool, giving families a way to record their experiences. For more details, see (Colineau and Paris, 2010).

\subsection{A family vs. individual spaces}

One of our goals was to explore whether providing a family space would be more conducive to engaging the whole family than having individual spaces for each family member. We implemented two versions of the portal. In one version, the portal was shared within the family: family members could see what the other members of the family had done, for example, their lifestyle ideas and their diary entries. In the other version, each individual in the family was given his or her own space; family members could only see their own entries. We tested both spaces with families, allocating them to either the shared or the individual version of the portal.

\subsubsection{Lifestyle ideas tool}

The lifestyle ideas tool was designed to help individuals reflect on their behaviour and to create opportunities for the family to talk about living more healthily. Two versions of the tool were implemented; one for each version of the portalshared vs. individual. The shared version allowed family members to see and browse all suggestions submitted (see Figure 1 left), and the individual version was specific to each member (see Figure 1 right). To submit a lifestyle change idea, people 
(ii)

Registration proper: If eligible, families were given more information to allow them to discuss the trial before signing up.

\subsubsection{Participants}

Our sample was represented by eighteen families with a mean age of $42.5(S D=6.67)$ years for the adults and $13.3(S D=1.37)$ years for the children. Amongst the children, there were slightly more girls than boys (55.5 per cent vs. 44.5 per cent respectively). Table 1 presents the proportion of overweight and obese adults and children in this sample. Adults were considered as overweight when their Body Mass Index (BMI) was between 25 and 29.9, and as obese when their BMI was over 30. For children, their BMI had to be compared with age and gender percentile charts. We did not ask participants for their social-economic status. Participants provided information about their Internet literacy (from basic" to advanced"). Most of them declared going online and frequently searching the web, but only a few engaged in social media activities (e.g. blogs, social networking).

Table 1: Distribution of overweight/obese individuals

\begin{tabular}{|c|c|c|c|}
\hline & $\begin{array}{l}\text { Total } \\
n(\%)\end{array}$ & $\begin{array}{l}\text { Shared } \\
\text { condition }\end{array}$ & $\begin{array}{l}\text { Individual } \\
\text { condition }\end{array}$ \\
\hline \multicolumn{4}{|l|}{ Overweight $(25 \leq \mathrm{BMI}<30)$} \\
\hline Father & $9(50)$ & $7(87.5)$ & $2(25)$ \\
\hline Mother & $4(22)$ & $2(25)$ & $2(25)$ \\
\hline Boys & 1 (6) & $1(12.5)$ & - \\
\hline Girls & $2(10)$ & $1(12.5)$ & $1(12.5)$ \\
\hline \multicolumn{4}{|l|}{ Obese ( $30 \leq \mathrm{BMI})$} \\
\hline Father & $8(44)$ & $2(25)$ & $6(75)$ \\
\hline Mother & $9(50)$ & $3(37.5)$ & $6(75)$ \\
\hline Boys & 5 (31) & $2(25)$ & $3(37.5)$ \\
\hline Girls & 1 (5) & - & $1(12.5)$ \\
\hline
\end{tabular}

\subsubsection{Task and goal}

Participating families were asked to look at their current lifestyle and provide suggestions to change it using the lifestyle ideas tool. Suggestions/ideas could be on any aspect of healthy living, including diet, exercise, alcohol consumption, sleep or more general wellbeing. We were not asking participants to change their behaviour, but rather to think about potential changes. According to the goal setting theory, providing an explicit goal leads to better performance than asking people to do their best (Locke and Latham, 2002). We thus gave families a goal: an explicit number of ideas to provide over the course of the trial. For some families, the goal (60 suggestions) was given at the family level and thus to be achieved by the family as a whole. In contrast, in other families, each family member was asked to provide 15 suggestions. In that case, there was no collective goal at the family level, but rather a set of individual goals. To determine an appropriate level of task difficulty, we conducted a self-efficacy test during the recruitment process with the participants registering their family. Based on the result of this test, we chose the target number of suggestions such that the task was perceived as feasible and yet moderately difficult.

\subsubsection{Procedure}

Families were randomly assigned to one of the conditions (shared or individual). In the shared condition, families were provided with a shared space (i.e. interacting with the shared version of the tools) and were given a collective goal to be achieved by the family as a whole. In the individual condition, family members were provided with an individual space (i.e. interacting with the individual version of the tools) and were given an individual goal to achieve.

During the recruitment process we contacted a large number of families, and 29 families were initially allocated to each condition. However, we were not able to retain all the families. Out of the 29 families allocated to the shared condition, 14 families did not visit the portal at all, two families withdrew for personal reasons, and we had to drop four families who came to the portal only once, logging in and out without doing anything. This left us with nine families (i.e. 36 family members). Similarly, in the individual condition, seven families did not visit the portal at all, one family withdrew for personal reasons, and we had to drop 12 families. This left us again with nine families. In these families, all family members participated. This represented an overall participation rate of 31 per cent, which is similar to attrition rates observed in internet-based interventions (Eysenbach, 2005). This provided us with enough data to examine trends, but not necessarily to have statistically significant results.

Two days before the start of the study, we e-mailed families a set of instructions with their task. At the end of the study, each family member had to complete a questionnaire. We then sent families a letter of appreciation along with a voucher to thank them. The voucher was not dependent on goal attainment.

\subsection{Results}

We looked at the different behaviours in the two conditions. In particular, we examined the number of lifestyle ideas submitted, whether individuals or families achieved their goal and how family members contributed to the task. We also explored the use of the diary tool and analysed the pattern of visits to the portal. 


\subsubsection{Use of the lifestyle ideas tool}

During the trial, participating families submitted a total of 792 lifestyle change ideas, with 312 suggestions submitted in the shared condition and 480 submitted in the individual condition. When reflecting on their lifestyle, families could look at it from a variety of perspectives, including for example getting more sleep, decreasing alcohol and/or cigarette consumption, eating regularly or complying with medications. A large majority of lifestyle ideas submitted were related to diet (41 per cent), physical activity (22 per cent) - e.g. having such a busy day at work didn't have enough time to have lunch. [...] i should have stopped working and walk down to healthshop to get a fresh fruit punch." (a 54 year old father), and health and wellbeing in general, including considerations such as regularity of meal, compliance to medication and quality time with the family (22 per cent) - e.g. Foo much TV. Skip a couple of shows and spend time with the family" (a 49 year old father), + often snack throughout the day instead of eating proper meals. Make sure that I eat regular small meals throughout the day." (a 33 year old mother). Other suggestions were related to sleeping habits (9 per cent) - e.g. it is late at night, and i'm still hooked on the computer game. Hope $i$ can get up tomorrow in time. stop playing when Mum asked me and go to bed" (a 16 year old boy); and also to smoking, stress, alcohol consumption and body image (7 per cent). (All comments from our participants are reproduced verbatim.)

Families with a collective goal and shared space submitted fewer lifestyle ideas (with an average of 34 submissions) than families in which individuals had an individual goal (with an average of 53). A between-within analysis of variance ${ }^{1}$ revealed that there was not enough evidence to establish a statistically significant difference between the two conditions $(F(1,16)=3.60, P=.076$ with $\alpha$ set at $.05)$. However, as the p-value is relatively low, the result suggests a trend in favour of the individual condition. In particular, we observed that, in the individual condition, (1) more families met their goal, and (2) there were less variability in the number of lifestyle ideas suggested by each family member. There could be several reasons for this.

First, as indicated by the goal setting theory, "goal specificity does reduce variation in performance by reducing the ambiguity about what is to be attained" (Locke and Latham, 2002). Although a specific goal was given in both the collective goal group and the individual goal group, it was not

${ }^{1}$ The family was the sampling unit and each family member was a repeated measure on this unit. Therefore, the between-subjects factor was the condition or group to which families were assigned (individual vs. shared), and the within-subjects factor was the type of family member (i.e. father, mother, oldest and youngest child). equally specific in both cases. Based on the answers to the post-task questionnaire administrated at the end of the study, it seems that the individual goal appeared to be clearer to family members as to what they had to do, and thus made them more accountable with respect to their participation, whereas the collective goal was seen as more imprecise, giving room for interpretation or negotiation as to who was to do how much. The participants' report suggested that no one in the family had a consistent view about what the family strategy was to achieve the collective goal. In contrast, the individual goal assigned to each member seems to have directed their individual effort and motivated them.

Second, it seems (based on the consistency of the individuals' performance) that family members were less committed to an overall collective goal than to an individual goal set by the experiment. This came as a bit of a surprise to us. By allowing the family to discuss the collective goal, and, in particular, to discuss and negotiate how each member would contribute (thus having each member participate in the setting of their own goal), we were expecting them to take ownership of the goal, and thus to be more committed to its attainment. It seems that the collective goal set for the family as a whole was more important for mothers, and that they tried to compensate for their family by contributing more.

Table 2: Lifestyle suggestions per family member

\begin{tabular}{|c|c|c|c|c|}
\hline & Fathers & Mothets & $\begin{array}{l}\text { Oldest } \\
\text { children }\end{array}$ & $\begin{array}{l}\text { Youngest } \\
\text { childen }\end{array}$ \\
\hline \multicolumn{5}{|l|}{ Family condition } \\
\hline Sum per tnember & 74 & 90 & 75 & 73 \\
\hline $\begin{array}{l}\text { Max number of } \\
\text { lifestyle ideas }\end{array}$ & 15 & 21 & 15 & 15 \\
\hline \multicolumn{5}{|l|}{ Individual condition } \\
\hline Sum per member & 120 & 120 & 121 & 119 \\
\hline $\begin{array}{l}\text { Max number of } \\
\text { lifestyle ideas }\end{array}$ & 16 & 16 & 17 & 16 \\
\hline Grand Total & 194 & 210 & 196 & 192 \\
\hline
\end{tabular}

To analyse the results further, we looked at the number of contributions per family member (see Table 2). It seems that, in the individual condition, all individuals have a fairly consistent output (about 13 submissions per member). This is consistent across all the families in this group. In contrast, in the shared condition, we observe a difference between the mothers and the rest of the family. Whereas fathers and children tend to contribute at a similar level (with an average of eight submissions each), mothers tend to do more with an average of ten suggestions. We investigated whether this difference was significant and it was not. The analysis of variance revealed that mothers did not contribute more than the rest of the family 
across conditions, and furthermore, that mothers' contributions did not depend upon the condition to which they were assigned (i.e. there were no effect of family member and no interaction).

We wondered whether task performance (i.e. number of lifestyle ideas provided) was related to the perceived degree of confidence individuals had about their ability (or the ability of their family) to accomplish the task. Table 3 shows the degree of confidence the person registering their family had in their family's ability to perform the task of submitting 60 lifestyle ideas (measured on a scale from 0 -annot do at all" to 100 highly certain can do"). We found that the perceived degree of confidence is not a good predictor of the performance of the family on the task. As highlighted in Table 3 (see boxed data), some were not confident at all, yet their family achieved their goal (60 suggestions), and themselves contributed fairly. In contrast, others, although quite confident, submitted only a few suggestions, and their family submitted very little as a whole. Interestingly, though, the applicants' individual scores seem a good predictor of the family performance. This suggests that the registrant may have been considered as the family leader in the task.

Table 3: Confidence score and performance for individuals and families in family condition

\begin{tabular}{|c|c|c|c|c|}
\hline \multicolumn{2}{|c|}{ Applicants } & \multirow{2}{*}{$\begin{array}{l}\text { Degree of confidence } \\
\text { for } 60 \text { suggestions }\end{array}$} & \multirow{2}{*}{$\begin{array}{l}\text { Individual } \\
\text { score }\end{array}$} & \multirow{2}{*}{$\begin{array}{l}\text { Family } \\
\text { score }\end{array}$} \\
\hline Family ID & Gender & & & \\
\hline 42 & $M$ & 0 & 7 & 42 \\
\hline 139 & $\mathrm{~F}$ & 0 & 15 & 60 \\
\hline 152 & $F$ & 10 & 1 & 4 \\
\hline 175 & $F$ & 10 & 10 & 39 \\
\hline 205 & $\mathrm{~F}$ & 20 & 3 & 12 \\
\hline 87 & $F$ & 60 & 3 & 11 \\
\hline 93 & M & 90 & 13 & 60 \\
\hline 78 & $F$ & 100 & 4 & 24 \\
\hline 166 & F & 100 & 19 & 60 \\
\hline
\end{tabular}

For the individual condition, there seems to be no relationship between the degree of confidence registrants had in being able to perform the task and their actual performance. No matter how confident they were, they all (except one) met their goal.

\subsubsection{Use of the diary tool}

We looked at the diary entries in the two conditions. Using the diary tool was not part of the task, and not everyone chose to take advantage of it. We were interested to see if it would be a useful tool for the family, who would use it and what type of memories people would be willing to record. Nine families (i.e. 26 individuals) out of the eighteen participating families used the diary tool to record their experience. A total of 222 diary entries were posted over the two week period (i.e. 58 diary entries in the shared condition vs. 164 in the individual condition). Families could choose over eight categories to record their experience. Table 4 shows the distribution of the entries per category. Families reported mainly about their intention to change, the changes that they made and what they implemented successfully.

Table 4: Number of diary entries per category

\begin{tabular}{|c|c|c|c|}
\hline & & $\begin{array}{l}\text { Family } \\
\text { condition }\end{array}$ & $\begin{array}{l}\text { Individual } \\
\text { condition }\end{array}$ \\
\hline Code & Entry Categories & $n(\%)$ & $n(\%)$ \\
\hline 1 & What I would consider giving a go & $16(28)$ & $57(35)$ \\
\hline 2 & What I tried successfully & $10(17)$ & $41(25)$ \\
\hline 3 & Changes that I made & $9(16)$ & $18(11)$ \\
\hline 4 & Readings I found interesting & $3(5)$ & $12(7)$ \\
\hline 5 & Tips and Strategies I found useful & $9(16)$ & $10(6)$ \\
\hline 6 & What I found challenging & $7(12)$ & $15(9)$ \\
\hline 7 & What did not work for me & $1(2)$ & $6(4)$ \\
\hline 8 & What would have helped me & $3(5)$ & $5(3)$ \\
\hline Total & & 58 & 164 \\
\hline
\end{tabular}

Here are examples of entries for each category: What I would consider giving a go- + would consider giving yoga a go and try and get friends involved. I am also going to try bite size snacks of fruit and veg my mum has made up in my fridge" (a 14 year old girl);

What I tried successfully- + have been successful with my plan to cut down on the amount of wine I drink of an evening. By not having any with my meal, and only one or two glasses while watching tv I have about halved the amount of alcohol I am having." (a 46 year old mother);

Changes that I made-eating more breakfast and going to bed earlier" (a 15 year old boy);

Readings I found interesting- + found the article about variety and serve sizes of foods interesting. It is sometimes hard to know just what constitutes a serve of fruit and veg." (a 46 year old mother);

Tips and Strategies I found useful- The tips about asthma friendly homes are really good and the idea about going to the doctor was something mum has wanted for a while but $i$ suppose $i$ had to be ready and reading it in black and white has made me want to go to the drs for an asthma plan." (a 14 year old girl);

What I found challenging-Fhe thing $i$ found most difficult was not eating lollies, that was so painfully hard." (a 13 year old girl); 
What did not work for me-- not snacking so much" (a 15 year old boy);

What would have helped me-It would have helped me to slow down and take these ideas on board one at a time instead of trying to do it all at once" (a 16 year old girl).

Although not all families took advantage of their electronic diary, when they did, it was generally used by the whole family (or at least 3 out of 4 members). The diary was mostly used by mothers and young girls (with a ratio of 1 to 2 ). While more mothers wrote in their dairy, we noted that it was also popular with the younger children who entered as many diary entries as mothers. When considering the entries by each family member, we observed that the use of the diary tool varied from one family member to another depending on the setting (shared with family vs. individual and private). Fathers, in particular, exhibited a different behaviour when in the shared $v s$. individual conditions with a number of diary entries 12 -fold higher in the individual condition. Indeed, when in the shared condition where all diary entries were shared among the family, fathers only submitted a couple of entries, whereas they submitted more diary entries than the rest of the family in the individual condition. This could suggest that fathers need more privacy to open up and reflect on their experience. In contrast, mothers and children submitted only two to three times more diary entries in the individual condition. In each condition, mothers and children had a similar level of engagement, with younger children submitting the most entries in the shared condition and older children submitting the least in the individual condition.

We investigated whether the use of the diary by each family member was moderated by the condition to which they were assigned. The between-within analysis of variance did not reveal any interaction effect. We did the analysis twice, including the whole sample (i.e. based on the 18 families) and excluding the families who did not use the diary tool. In both cases, there was not enough evidence to show any statistical differences. There was no main effect of family members and not enough evidence of group effect $(F(1,7)=3.56$, $P=.10$; based on families who used the tool).

While the task was set up to encourage families to critically review their health behaviour and discuss it together, we were interested to see whether we could capture in the diary entries any sign of change in attitude, and whether families and/or individuals will start implementing some of the changes they proposed. In line with the lifestyle change ideas submitted, families mostly reported occasions when they successfully included in their daily routine a walk, used their bike or switched for healthier lunch options. For some of them, it seems that the positive experience of the trial could encourage them to more long-lasting change: The long walk in the evening helped me to relax and partially suppressed my appetite. My mood tends to be less frustrated also - definitely an improvement in mood. The thought of more regular exercise after work could be a viable option" (a 42 year old mother); +think walking alot today was a really good option for me and $i$ have realised now that $i$ should probably walk to and from school everyday. I think $i$ might try this out starting on monday." (a 13 year old boy).

Besides successful trials, families also reported changes that they made to their lifestyle. It was interesting to see that encouraging and facilitating reflective thinking (through the lifestyle and diary tools) could lead to change in attitude and maybe change in behaviour. Although families mostly reported trying to exercise more and eating more healthily, the changes implemented by individuals and families included a variety of health and wellbeing changes: Didn't feel so bad waking up to No Cigarettes this morning, there was just a pang of deprivation but nothing horrific - after all, I managed without them All Day yesterday. So that's what I'm doing, just getting on with it, ignoring the cravings." (a 53 year old mother); My body is finding it much easier to sleep with the new sleep tecniques that $i$ learnt on the healthy living website. It really is a good website and suggested that i may have sleep aponea so $i$ went to my $d r$ and am a prime candidate for a cpap machine. lifes looking up!' (a 44 year old mother).

Among the diary entries, we also observed some changes related to quality time spent with the family, as suggested by these entries: My eldest daughter and $i$ have made a pact to ride our bikes once a week and im sure it will bring us closer together and stop her nagging me for the last year to ride it." (a 42 year old father); +have made alot of changes this last week that will help my body including exercising, dieting reducing fat working on my self esteem and having more family time." (a 14 year girl). Finally, it is interesting to note that in the individual condition in particular, where each member was working independently towards an individual goal, there were several mentions in the diary of family discussions and supportive behaviours within the family: + have made alot of changes during this process including novel ways to exercise getting closer to my family because we are regularly discussing this and many ways to lose weight by dieting and trying new food." (a 42 year old father); $f . .$.$] Also my family and i$ sat down and had a chat about this survey and we have learnt alot about each other and are supporting each others plans." (a 44 year old mother). 
In the post-task questionnaire that families completed at the end of the study, we asked participants if they had tried some of their suggestions. As already reported by some people in their diary entries, we found that families tried and implemented lifestyle changes. In the shared condition, families implemented most of the changes with 83 per cent of participants reporting having tried a few (sometimes many) suggestions compared to 74 per cent in the individual condition. In both conditions, mothers were the most proactive member to implement the changes.

\subsubsection{Visits to the site: how often and what do people do?}

We examined the patterns of visits to the portal done by families during the trial. Although we observed some differences on a daily basis, there are essentially as many visits in each of the two conditions (i.e. 197 in the individual condition vs. 198 in the shared one). If anything, the pattern of visits seems to be more fairly distributed in the individual condition than in the family one, which is characterised by peaks of visits at the beginning and the end of the study. We also looked at the number of visits per family member. We observed differences between one family member to another, and also from one condition to the other. For example, fathers and older children visited the portal less often in the shared condition than in the individual one, whereas in contrast, mothers and younger children did more visits when in the shared condition.

There is one constant however. In both conditions, mothers tend to visit the portal more than the rest of the family. When checking whether the number of visits done by each family member was moderated by the condition to which they were assigned, the analysis of variance did not show any interaction effect. There was no main effect of group either. However, we found evidence of main effect for family members. Contrast testing indicated that mothers across the two conditions did on average more visits to the portal than the rest of the family, a statistically significant result $(F(1,16)=17.59, P \leq .001)$. There were no significant differences between the fathers and the children, or between the oldest and youngest children.

Among the total number of visits to the portal, we looked at the number of visits that were related to the task compared to those which were not. We define a task-related visit as a visit during which participants submitted lifestyle suggestions. In the shared condition, we observed a total of 145 taskrelated visits. This means that 53 visits were not task-related. This represents about 26.8 per cent of the total number of visits to the portal. In contrast, families in the individual condition did a total of 170 task-related visits. There were 27 visits not taskrelated in this condition, which represents only 13.7 per cent of the total number of visits. This suggests that, in the shared condition, participants came to the portal for reasons other than to perform the task.

We examined further the visits to the portal that were not related to performing the task. We wanted to understand who did these visits (which particular individual in the family) and what motivated these visits. Children seemed to be particularly keen to visit the portal (with a total of 25 visits and 18 for older and younger children respectively), as were mothers, whereas fathers were the family member the least involved. The figures for the non task-related visits indicate that some people did only task-related visits to the portal whereas others did up to 8 additional visits.

We turned to the diary tool and the resource section of the portal to try to understand the difference in the number of visits. As most of the diary entries were submitted in the individual condition, this may explain the non task-related visits observed in this condition. However, it does not explain the difference observed in non taskrelated visits between the two conditions. To understand what motivated families to come to the portal in addition to performing their task, we examined the use of the health resources. We looked at the patterns of participants checking the available resources in both conditions, and there were essentially no difference between the groups. In both cases, 16 users out of 36 browsed the health section, and most of them (15 out of the 16) visited a web page.

One of the main differences between the two conditions was that, in one condition, the space was shared, allowing members of the family to see and read what others had posted, whereas in the other condition, the space was more personal and individually oriented. Therefore a possible reason for participants assigned to the shared condition to do additional visits to the portal was that they were interested to read about other family members' suggestions and learn about their experience. If that is the case, the shared condition might have encouraged more communication and support amongst family members, potentially also encouraging them to discuss ideas and issues further within the family.

\section{DISCUSSION}

Both environments (shared vs. individual) had their strengths and weaknesses. On the one hand, it seems that providing an individual space has encouraged personal reflection with a large number 
of diary entries submitted by individuals. Although we were unable to demonstrate statistical significance, fathers, in particular, seemed to have taken advantages of the personal diary. As fathers are often reported as the most difficult family member with whom to engage, this may be a way to motivate them and get their commitment. It appears also that giving specific goals at the individual level results in better performances in terms of a task. Family members working towards an individual goal seemed keener to visit the portal regularly.

On the other hand, it appears that encouraging the family to work together towards a collective goal has promoted support within the family. Based on answers from the post-task questionnaire, we learnt that 1 in 2 family member received help in the shared condition against 1 in 4 in the individual one, with family members helping each other in thinking of what to change and how to change their lifestyle. One of our objectives was to help families work together to achieve a healthier lifestyle, so in that respect the shared condition is promising even if it was less successful in terms of task accomplishment. In addition, the shared environment seems to have encouraged more changes with more people trying some of their suggestions and starting to implement them in their daily routine. This may have been facilitated by the opportunity for members to look at what others were suggesting and reporting.

Interestingly, both environments supported health discussions within the family and outside the family. In the shared condition, participants reported discussing the task mostly with someone in their family (75 per cent) whereas in the individual condition, participants reported discussing the task together as a family ( 91 per cent). This may have been a way for families in the individual condition to compensate for the fact that they had separate spaces.

Our findings suggest that it is desirable to enable people to personally reflect on their lifestyle and the changes they could make, thus giving them specific individual goals. While writing exercises have been shown to provide some health benefits, our results further suggest that providing an environment for people to reflect on their health behaviour may lead to a positive health attitude and encourage health behaviour changes. At the same time, it is important to design an environment that enables family members to share their thoughts with the rest of the family and encourage the family to discuss together potential changes and support each other in implementing these changes. While mothers are typically the most proactive, it is still possible to engage the other members of the family, as evidenced by our study.
The two week study turned out to be a pleasant learning experience for most of the participating families, and overall we got very positive feedback, as illustrated in the following comments: +enjoyed taking part in this survey as it opened my eyes to just how much difference $i$ can make to the healthy lifestyle of myself and my family by just making a few small choices" (a 40 year old mother). + currently have no healthy role models to look up to as our whole family is in need of help. Now that we have started this survey $i$ can look up to my mum and dad as they are trying real hard to be healthier by exercising, eating and sleeping right and being there for us for support." (a 16 year old girl).

\section{CONCLUSION}

Our research aims at helping families work together to achieve a healthier lifestyle, increasing their awareness of what they currently do and what they could do differently. We built a prototype collaborative platform for families to see if we could facilitate such discussions and encourage supportive behaviour within the family. We investigated in particular whether it would be more promising to have an individual profile, space and goal, for each individual in a family, or a shared profile, space and goal. Based on the results of the two week trial we conducted, we argue that both individual and shared profiles have their strengths and weaknesses and brought some interesting results. The choice of one (or elements of one) vs. the other depends very much on one's targeted outcome. In this study, we were interested in helping families work together to achieve a healthier lifestyle. Setting a collective goal for the family seemed to have promoted support within the family, so in that respect the shared condition brought promising results even if it was less successful in terms of task accomplishment. We were also interested in encouraging families to reflect on their health behaviour. The individual space seems to have encouraged more personal reflections as measured by the number of diary entries, suggesting that private space may be important as well. In both conditions, parents and children were able to reflect on their lifestyle, suggest changes and even implement them, suggesting that engaging the whole family can be a promising way forward. Our study also provided evidence that technology can play a role in supporting reflection and sharing within the family context, which was not obvious at the start, considering that many interactions within a family take place offline.

In the study, we tested the impact of individual goal vs. collective goal, and individual space vs. shared space. Although we tested them by associating the individual goal with an individual space and a 
collective goal with a shared space, it might be interesting to test other combinations based on what we learnt. We now intend to apply these ideas in the next version of the prototype, providing both a shared space to encourage discussions and support, potentially leading to changes, and individual spaces and goals to promote reflection and commitment to the goal.

\section{ACKNOWLEDGMENTS}

We would like to thank Greg Smith for developing the family portal and lan Coombe and Dipak Bhandari for helping us in recruiting families and corresponding with them during the period of the study. We also thank all the people who participated in our study.

This work has been jointly funded by the CSIROled Preventative Health National Research Flagship, the Australian Government through the Intelligent Island Program and the CSIRO. The Intelligent Island Program is administered by the Tasmanian Department of Economic Development, Tourism, and the Arts.

\section{REFERENCES}

Brown, B., Chetty, M., Grimes, A. and Harmon E. (2006). Reflecting on Health: A system for students to monitor diet and exercise. In the Proceedings of CHI 2006, Montreal, Canada, April 22-27, pp. 1807-1812.

Colineau, N., Paris, C., Marendy, P., Bhandari, D. and Shu, Y. (2009). Family Engagement in Weight Management. In CHI 2009, Extended Abstract Boston, MA, USA, April 3-9, pp. 3991-3996.

Colineau, N. and Paris, C. (2010). A Portal to Promote Healthy Living within Families. In the 5th International Workshop on Personalisation for eHealth held in conjunction with the eHealth 2010 Conference. Casablanca, Morocco, Dec. 13, 2010. Consolvo, S., McDonald. D.W., Toscos, T., Chen, M.Y., Froehlich, J., Harrison, B., Klasnja, P., LaMarca, A., LeGrand, L., Libby, R., Smith, I. and Landay, J.A. (2008). Activity sensing in the wild: a field trial of UbiFit garden. In the Proceedings of CHI 2008, Florence, Italy, April 05-10, pp. 17971806.

De Bourdeaudhuij, I. (1997). Perceived family members' influence on introducing healthy food into the family. Health Educ. Res. 12(1):77-90.

Eysenbach, G. (2005). The Law of Attrition. J. Med. Internet Res. 7(1):e11

available online: $w w w . j m i r . o r g / 2005 / 1 / e 11 /$
Grimes, A., Bednar, M., Bolter, J.D. and Grinter, R.E. (2008). EatWell: Sharing Nutrition-Related Memories in a Low-Income community. In Proceedings of CSCW 2008, San Diego, CA, USA, Nov. 8-12, pp. 87-96.

Grimes, A., Desney, T. and Morris, D. (2009). Toward technologies that support family reflections on health. In ACM 2009 Group Conference, Sanibel Island, FL, USA, May 10-13, pp. 311-320. Hardeman, W., Griffin, S., Johnston, M., Kinmonth, A.L., Wareham, N.J. (2000). Interventions to prevent weight gain: a systematic review of psychological models and behaviour change methods. Int. J. Obes. 24:131-143.

Jones, D., Beach, S. and Jackson, H. (2004). Family influences on Health: A framework to organize research and guide intervention. Handbook of Family Communication. Lawrence Erlbaum Assoc., Mahwah NJ, pp. 647-672.

Locke, E.A., Latham, G. P. (2002). Building a Practically Useful Theory of Goal Setting and Task Motivation. Am. Psychol. 57(9):705-717.

Mamykina, L., Mynatt, E.D., Davidson, P.R., and Greenblatt, D. (2008). MAHI: Investigation of Social Scaffolding for Reflective Thinking in Diabetes Management. In the Proceedings of CHI 2008, Florence, Italy, April 05-10, pp. 477-486.

Mattila, E., Pärkkä, J., Hermersdorf, M., Kaasinen, J., Vainio, J., Samposalo, K., Merilahti, J., Kolari, J., Kulju, M., Lappalainen, R. and Korhonen, I. (200). Mobile Diary for Wellness ManagementResults on Usage and Usability in Two User Studies. IEEE Trans. Inf. Technol. Biomed. 12(4):501-512.

McLean, N., Griffin, S., Toney, K. and Hardeman, W. (2003). Family Involvement in Weight Control, Weight Maintenance and Weight-loss Interventions: A Systematic Review of Randomised Trials. Int. J. Obes. 27:987-1005.

Noar, S.M., Benac, C.N. and Harris, M.S. (2007). Does Tailoring Matter? Meta-Analytic Review of Tailored Print Health Behaviour Change Interventions. Psychol. Bull. 133(4):673-693.

Toscos, T., Connelly, K. (2008). Encouraging Physical Activity in Teens: can technology help reduce barriers to physical activity in adolescent girls? In Pervasive Health 2008, Tampere, Finland, January 30-February 1.

Verheijden, M.W., Bakx, J.C., van Weel, C., Koelen, M.A. and van Staveren, W.A. (2005). Role of social support in lifestyle-focused weight management interventions. Eur. J. Clin. Nut. 59, Suppl 1, S179-S186. 